\title{
Serological Surveillance for Non-Rabies Lyssaviruses among Apparently Healthy Dogs in Zaria, Nigeria.
}

\author{
${ }^{1}$ DZIKWI*, A.A., ${ }^{1}$ UMOH, J.U., ${ }^{1}$ KWAGA, J.K.P., ${ }^{2}$ AHMAD, A.A.
}

1 Department of Veterinary Public Health and Preventive Medicine, Faculty of Veterinary Medicine, ABU Zaria.

${ }^{2}$ Department of Microbiology, Faculty of Science, ABU Zaria. *Corresponding Author.asabezik@yahoo.com.Tel: 08035912881.

\section{SUMMARY}

Out of the seven recognized and four putative genotypes of lyssaviruses, genotypes 1-3 comprising namely the classical rabies virus (RABV), Lagos bat virus (LBV) and Mokola virus (MOKV) respectively have been reported in Nigeria. The domestic dog, Canis familiaris, is recognized as the reservoir for genotype 1, and the straw-colored fruit bat for genotype 2. The reservoir for genotype 3 remains unidentified. Serum samples were collected by convenient sampling from apparently healthy dogs in Zaria and tested for the presence of neutralizing antibodies to three members of lyssavirus genotypes, namely LBV, MOKV , Duvenhage (DUVV), and a putative genotype, West Caucasian bat lyssavirus (WCBV) using a modification of the rapid fluorescent focus inhibition test (RFFIT). Out of the 189 samples tested, six (3.7\%) of them neutralized Lagos bat virus, and two (1.1\%) of these additionally showed a neutralizing activity against Mokola virus. There was no serological evidence of WCBV and DUVV. This finding suggests the presence of phylogroup 2 lyssaviruses circulating among dogs in this location. This is important especially since human exposure to dog bite is common in this locality. The possibility of exposure to these genotypes will have great implication with regards to the usefulness of the available vaccines which do not sufficiently protect against members of phylogroup 2. It further emphasizes the poor understanding of the epidemiology of lyssavirus infection, especially among apparently healthy dogs. This calls for enhanced surveillance for lyssaviruses among both domestic and wildlife species in Nigeria to identify the definitive reservoir for Mokola virus.

Keywords: Dogs, Lyssaviruses, Phylogroup 2, Zaria.

\section{INTRODUCTION}

Rabies is a viral zoonosis characterised by encephalomyelitis. It a disease of great public health concern because the case fatality rate is almost 100 percent (Rupprecht et al., 2002). The aetiologic agents of rabies are members of the family Rhabdoviridae, with seven recognised genotypes. The classical rabies virus (RABV, genotype 1) was the only known member, until 1956 when the Lagos bat virus (LBV, Genotype 2) was isolated, and this was followed by the isolation of Mokola virus (genotype 3) in 1968. LBV was first isolated from one of seven pooled samples of 42 brain tissues of the straw-coloured frugivorous bats, Eidolon helvum, on Lagos island (Boulger and Porterfield, 1958) and thereafter, several isolations were reported from other species of bats, and in cats and dogs across Africa (Kuzmin and Rupprecht, 2007) . The initial isolation of MOKV was from the viscera of shrews, Crocidura spp, in Mokola, Ibadan, Nigeria (Kemp et al., 1972). This was followed with isolations of the virus from other terrestrial mammals including domestic cats and dogs, other rodents and in man (Kuzmin and Rupprecht, 2007). Genotypes 4-7 include the Duvenhage virus (DUVV), European bat lyssaviruses 1 and 2 (EBV1 and 2) and the Australian bat lyssavirus (ABV) respectively. DUVV was isolated from a man in 1970 in South Africa and subsequently in bats, but never from terrestrial mammals. It is closely related to EBV and though an African isolate, it differs very much genotypically from LBV and MOKV. Four putative genotypes were isolated from bats namely Irkut (East Siberia), Kudjan and Aravan (Central Asia) and the West Caucasian bat virus (Caucasian region) are also recognised (WHO, 2005).

The lyssavirus genotypes have been placed into 2 phylogroups, with genotypes 1, 45, 6 
and 7 in phylogroup 1 and genotypes 2 and 3 in phylogroup 2 . The members of phylogroup 2 have to date been reported only on the African continent. WCBV shows a limited phylogenetic relatedness to LBV and MOKV (Kuzmin and Rupprecht, 2007).

To date, human infection has not been reported from LBV, but infection with MOKV has been reported in two persons in Nigeria in 1968 and 1971 (Shope et al., 1970; Familusi et al., 1972; Kemp et al., 1972). The domestic dog is the established reservoir for RABV in Nigeria and the virus is maintained in this host. The definitive host for LBV is the fruit bat, while that of MOKV has not yet been identified but speculations abound of the possibility of insectivores, rodents and possibly bats serving to maintain it (Niezgoda et al., 2002: Nadin-Davis, 2007). Effective and economical methods for rabies control exist but the disease remains neglected in many countries (Knobel et al., 2005)

\section{MATERIALS AND METHODS Collection and Handling of Samples}

Serum samples were collected from 200 apparently healthy dogs from locations in Zaria namely: Sabon Gari, Samaru, and Ahmadu Bello University Zaria Staff Quarters. The dogs were selected using convenient sampling, and permission for the study was sought from the heads of the wards included in the study. This was followed by visits to households owning dogs and consent for the study sought from the adult members of the homes. A family member i.e. the person responsible for the dog was interviewed and a questionnaire filled out. Dogs were clinically examined for health status by a veterinarian and only those that appeared healthy were included in the study. Information obtained included age, sex, and vaccination history of the dogs. Approximately $5 \mathrm{mls}$ of blood was collected from the cephalic veins of the dogs using sterile syringe and needle, and transferred to universal bottles. The specimens were transported to the Viral Zoonoses Laboratory, Department of Veterinary Public Health and Preventive Medicine, A.B.U Zaria. The blood samples were then allowed to stand in a slanting position to clot and serum samples were harvested using Pasteur pipettes. Sera were stored in individual tubes at $-20^{\circ} \mathrm{C}$. The samples were transported (according to the international standards for the transportation of infectious agents, category A) on dry ice to the Rabies Laboratory of the Centre for Disease Control and Prevention (CDC), Atlanta, Georgia, for analysis.

\section{Laboratory Analysis}

A modified rapid fluorescent focus inhibition test (RFFIT) was used for testing of sera for neutralizing antibodies to LBV, MOKV DUVV and WCBV. The analyses were performed according to the Standard Operating Procedure (CDC RFFIT SOP, 2006). Briefly described, the reaction was performed using 4-well $(6 \mathrm{~mm})$ glass slides (Cel-Line, Erie Scientific, Portsmouth, New Hampshire, USA). Sera were preheated at$56^{\circ} \mathrm{C}$ for inactivation. Heat inactivated serum samples were tested initially in 1:10 and 1: 25 dilutions. Approximately $14 \mu \mathrm{l}$ minimum essential medium (MEM)-10 was added to the $1^{\text {st }}$ and $3^{\text {rd }}$ well of 4-well Tefloncoated slides, and $7.5 \mu \mathrm{l}$ of the same medium was dispensed into the $2^{\text {nd }}$ and $4^{\text {th }}$ well. Approximately $3.6 \mu \mathrm{l}$ of two separate test sera were added to the $1^{\text {st }}$ and $3^{\text {rd }}$ wells respectively, and the contents of each well mixed properly using the pipette tip. Five microliters of the mixture was then transferred to corresponding $2^{\text {nd }}$ and $4^{\text {th }}$ well and mixed properly using tip of the micropipette.

LBV (isolate from Nigeria, 1956), MOKV (isolate from South Africa, 1998), DUVV (isolate from South Africa, 1970), and WCBV (isolate from Russia, 2002) were used for the reaction. About $50 \mathrm{FFD}_{50}$ (fluorescing foci doses) of each virus was used as confirmed by a titration on a control slide with each reaction run. The slides were incubated in a humidity chamber at $37^{\circ} \mathrm{C}$ for 90 minutes. Thereafter, $25 \mu \mathrm{l}$ of mouse neuroblastoma cells (MNA) were added (concentration approximately $2 \times 10^{6}$ cells $/ \mathrm{ml}$ ), and the slides were incubated at $37^{\circ} \mathrm{C}$ for 20 hours with MOKV and 44 hours for LBV, WCB, and DUVV. Slides were then 
fixed in cold acetone for 30 minutes, air dried and subjected to the DFA test as described by Dean et al. (1996). At microscopy, 10 separate fields were counted for each well. For all positive or inconclusive RFFIT results, additional titration of such sera was conducted in dilutions $1: 10,1: 25$, $1: 625$ and $1: 15625$, to validate the confirmation of positive result. The titers were calculated using Reed and Muench method (1938) and expressed as log dilution of the test serum. Only the samples that had a $50 \%$ end-point neutralizing titre greater than $1 \log _{10}$ (e.g. less than 5 fields contained infected cells at serum dilution 1:10) were considered as positive. remaining 11 were cytotoxic and could not be tested. Six (3.2\%) of the dog sera neutralized LBV and two (1.1\%) additionally showed neutralizing activity to MOKV (Table 1). Though, records have it that $5 / 6(83 \%)$ of these dogs have been vaccinated against rabies, there is no expectation of cross-reactivity between the virus neutralizing antibodies (VNA) to rabies and these genotypes. All the dogs that had VNAs to these lyssaviruses were adults and majority (83\%) was male (Table I). The only female that was positive happened to be previously unvaccinated and was one of the two that showed neutralizing activity to both LBV and MOKV.

\section{RESULTS}

A total of 189 samples were tested. The

TABLE I. Biodata and virus neutralizing antibody titers to Lagos bat virus and Mokola virus in apparently healthy dogs in Zaria.

\begin{tabular}{|llllll|}
\hline Dog ID \# & LBV VNA titer & MOKV VNA titer & Age & Sex & ${ }^{*}$ Vaccinationstatus \\
\hline A3/26 & 1.51 & 1.07 & Adult & Male & Vaccinated \\
DS/33 & 1.48 & 1.28 & Adult & Female & Unvaccinated \\
C71 & 1.22 & & Adult & Male & Vaccinated \\
C4 & 1.07 & & Adult & Male & Vaccinated \\
Q2/13 & 1.25 & & Adult & Male & Vaccinated \\
C65 & 1.11 & & Adult & Male & Vaccinated \\
AVERAGE & 1.27 & 1.18 & & & \\
\hline
\end{tabular}

* Vaccination status refers to information provided by owners about previous vaccination of the dogs against rabies (genotype 1).

\section{DISCUSSION}

Cross-neutralising activity between LBV and MOKV occurs and may explain the occurrence in this study (Hanlon et al., 2005: Dzikwi et al., 2010). Another possible

explanation is concurrent infection with the two lyssaviruses in those two animals since not all the six LBV positive sera additionally neutralised the MOKV. The average VNA titers for the two viruses were approximately the same. This suggests a low level of circulation of the virus. Similar findings of VNA to LBV and MOKV has been reported in the past in Nigeria (Ogunkoya et al., 1990) in which $5.8 \%$ of tested animals had VNA to LBV and $17.4 \%$ to MOKV but in the present study, the percentages were lower $(3.7 \%$ and $1.1 \%$ for LBV and MOKV respectively). There is a large population of dogs in the study area, many of which are free-roaming. The poor dog ownership practise in this region allows dogs to scavenge for their food (Adaba et al., 2004). It is possible that the dogs probably fed on carcasses of some smaller mammals like rodents or even bats which may have been infected with the MOKV.

MOKV is very poorly understood with regards to its ecology, circulation and other properties that explain its epidemiology (Kuzmin and Rupprecht, 2007). While the reservoir of LBV is established to be the 
frugivorous bats, that of MOKV remains unknown. Some author have speculated the possibility of rodents and bats as possible reservoirs of MOKV (Nadin-Davis, 2007), especially that the virus was first isolated in wild rodents in Nigeria (Shope et al., 1970). Lyssavirus members of the phylogroup 2 are important because with their presence, the epidemiology of rabies is modified. The implication is great for the control of lyssaviruses in Nigeria. Rabies vaccines that are currently used do not have crossneutralizing activity against members of phylogroup 2 (Nel, 2005).

There was no neutralising activity against WCBV and DUVV from this study. WCBV was included because it has shown some phylogenetic relatedness to members of phylogroup 2 (Kuzmin and Rupprecht, 2007). This virus was isolated from bats in the Caucasian region and has not been reported in any other part of the world. DUVV is more related to RABV than phylogroup 2 members. From this study, the non-rabies lyssaviruses circulating among apparently healthy dogs in Nigeria remain LBV and MOKV.

Human exposure to dog bite is very common in this region. If the presence of these antibodies is as a result of infection, then there is the danger of potential exposure to these viruses following dog bites. The implication of having members of phylogroup 2 circulating in the study area is considerable since the available vaccine can only protect against members of phylogroup 1 to which these two (LBV and MOKV) do not belong. Being in their own class and with our limited understanding of their epidemiology, extensive surveillance needs to be carried out among both domestic and wild animals to identify the reservoirs for MOKV and to provide information for development of vaccines that will effectively protect against infection with members of this phylogroup.

\section{ACKNOWLEDGEMENT}

The authors acknowledge the cooperation of the Rabies Laboratory of CDC, Atlanta Georgia for supporting this work and providing the materials and space for the work.

\section{REFERENCES}

ADABA, J.I., DZIKWI, A.A., and UMOH, J.U. (2004). Effect of dog ownership patterns on the utilisation of veterinary services: A case study of Sabon Gari local Government area of Kaduna State, Nigeria. Proceedings of the $41^{\text {st }}$ congress of the Nigerian Veterinary Medical Association. P 104-105.

OGUNKOYA, A.B., BERAN, G.W., UMOH, J.U., GOMWALK, N.E. and ABDULKADIR, I.A. (1990). Serological evidence of infection of dogs and man in Nigeria by lyssaviruses (family Rhabdoviridae). Transactions Royal Society for Tropical Medicine and Hygiene 84:842-845.

BOULGER, I.R., AND J.S. PORTERFEILD (1958).Isolation of a virus from Nigerian fruit bats. Transactions of the Royal Society of Tropical Medicine and Hygiene 52: 421-424.

DZIKWI, A.A., KUZMIN, I.V., UMOH, J.U., KWAGA, J.K.P., AHMAD, A.A., RUPPRECHT, C.E (2010). Evidence of Lagos bat virus circulation among Nigerian fruit bats. Journal of wildlife disease 46: 267-271.

SHOPE, R.E.,F.A. MURPHY, A.K. HARRISON, O.R. CAUSEY, G.E. KEMP, G.I.H. SIMPSON, AND D. MOORE. (1970). Two African viruses serologically and morphologically related to rabies virus. Journal of Virology 6: 690-692.

FAMILUSI, J.B., OSUNKOYA, B.O., MOORE, D.L., KEMP, G.E. AND FABIYI, A. (1972). A fatal human infection with Mokola virus. American Journal of Tropical Medicine and Hygiene 21:959-963.

KEMP, G.E., CAUSEY, O.R., MOORE, D.L., DELOLA, A. AND FABIYI, A. (1972). Mokola virus. Further studies on IBAN 27377, a new rabies-related etiologic agent of zoonosis in Nigeria. American Journal of Tropical Medicine and Hygiene 21:356-9

C.D.C. (2006). Standard Operating Procedure for the rapid fluorescent focus inhibition test (RFFIT) for 
determining rabies virus neutralizing antibody. Revised June, 2006. Pp 1-192

DEAN, D.J., ABELSETH, M.K, AND ATANASIU, P. (1996). The fluorescent antibody test In: Laboratory techniques in rabies, Meslin, F-X., Kaplan, M.M., Koprowski, H. (eds), $4^{\text {th }}$ edition. Geneva, Switzerland. World Health Organisation, 88-95.

HANLON, C.A., KUZMIN, I.V., BLANTON, J.D., WELDON, W.C., MANANGAN, J.S. AND RUPPRECHT, C.E. (2005). Efficacy of rabies biologics against new lyssaviruses from Eurasia. Virus Research 111:44-45.

KNOBEL, D.L., CLEAVELAND, S., COLEMAN, P.G., FEVRE, E.M., MELTZER, M.I., MIRANDA, M.E.G., SHAW, A., ZINSSTAG, J. AND MESLIN, F-X (2005). Re-evaluating the burden of rabies in Africa and Asia. Bulletin of the World Health Organisation, 83:360369.

KUZMIN, I.V AND RUPPRECHT, C.E (2007). Bat rabies. In Rabies ( $2^{\text {nd }}$ edition). Jackson, A.C and Wunner, W.H (editors). Elsevier Inc, London UK. 259307
NADIN-DAVIS, S.A (2007). Molecular Epidemiology. In Rabies ( ${ }^{\text {nd }}$ edition). Jackson, A.C and Wunner, W.H (editors). Elsevier Inc, London, UK. 69122.

NEL, L.H. (2005). Vaccines for lyssaviruses other than rabies. Experts Review Vaccines 4: 533-540

NIEZGODA, M., HANLON, C. A. AND RUPPRECHT, C.E. (2002). Animal rabies In: Rabies. Jackson, C.A., and Wunner, W.H (eds). Academic Press, San Diego, pp 163-218.

REED, L.J AND MUENCH, H (1938). A simple method of estimating fifty percent end-points. The American Journal of Hygiene 27: 493-497.

RUPPRECHT CE, HANLON CA, HEMACHUDA $T$ (2002). Rabies reexamined. Lancet Infect Dis.2:327-43. DOI: 10.1016/S1473-3099-(02)002876

W.H.O. (2005). WHO Expert Consultation on Rabies. WHO Technical Report series, 931. 